\title{
Influence of the settling of the resin beads on Diffusion Gradients in Thin films measurements
}

Martín Jiménez-Piedrahita ${ }^{\mathrm{a}}$, Alexandra Altier ${ }^{\mathrm{a}}$, Joan Cecilia ${ }^{\mathrm{b}}$, Carlos Rey-Castro ${ }^{\mathrm{a}}$, Josep

Galceran $^{\mathrm{a}}$ and Jaume Puy ${ }^{\mathrm{a} *}$

${ }^{\mathrm{a} D e p a r t a m e n t ~ d e ~ Q u i ́ m i c a, ~}{ }^{\mathrm{b}}$ Departament de Matemàtica, Universitat de Lleida and AGROTECNIO, Rovira Roure 191, 25198, Lleida, Spain

* Phone number 34973702529

email jpuy@quimica.udl.cat

\section{Abstract}

Binding resin beads used in DGT (Diffusion Gradients in Thin films) tend to settle to one side of the resin during casting. This phenomenon might be relevant for metal accumulation when partially labile complexes dominate the metal speciation, after recognizing the important role played by complex dissociation in the resin domain. The influence of the inhomogeneity of the binding agent distribution on metal accumulation is here assessed by numerical simulation of DGT devices with binding beads in only one half of the resin disc, as a reasonable model of the standard resin discs. Results indicate that a decrease in mass accumulation of less than $13 \%$ can arise in these inhomogeneous devices (as compared with an ideal disc with homogeneous dispersion of the resin beads) when complexes with stability constant $K<10^{2} \mathrm{~m}^{3} \mathrm{~mol}^{-1}\left(K<10^{5} \mathrm{~L} \mathrm{~mol}^{-1}\right)$ dominate the metal speciation. The loss increases as $K$ increases, but the percentage of mass loss always remains lower than the volume fraction of resin disc without beads. For very labile or inert complexes, the impact of the inhomogeneous distribution of binding resin beads is negligible. As kinetic 
dissociation constants of complexes can be estimated from the distribution of the metal accumulation in a DGT device with a stack of two resin discs, the influence of the inhomogeneity on the recovered kinetic constant is also assessed. For the cases studied, the recovered kinetic dissociation constant, $k_{\mathrm{d}, \text { recovered }}$, retains the correct order of magnitude, being related to the true $k_{\mathrm{d}}$ by $k_{\mathrm{d}} \approx f^{-1} k_{\mathrm{d} \text {,recovered, }}$ quite independently of $K$ and $k_{\mathrm{d}}$ values, being $f$ the fraction of volume of the resin disc where resin beads are dispersed.

\section{Introduction}

DGT devices were developed for the in situ measurement of trace metals in waters [1, 2]. They consist of two layers, the resin gel layer (which contains a strong binding agent to accumulate the metals (usually Chelex 100)) and the diffusive gel (which minimizes the influence of the hydrodynamic conditions in the sample solution on the metal accumulation). Both gel layers are covered by a filter, which separates them from the solution [3]. Extensions of these devices were developed for sediments and soils [4] and now, as a result of an intensive research work addressed to develop new binding agents, there are DGT devices for the accumulation of anions such us phosphate [5], non-metals and metalloids such As, Se or Sb species, other special cations such as $\mathrm{Hg}[6]$ or $\mathrm{K}[7,8]$ and priority polar or non-polar substances [9, 10]. Recently, different publications have highlighted the important role played by the resin disc thickness in determining the metal accumulation in systems with partially labile complexes [11-16]. The argument starts by assuming a fast and strong binding of the metal to the resin sites. Accordingly, the complexation equilibrium shifts towards dissociation, so that complexes release free metal in the gel domain which binds to the resin. In this way, the thickness of the reaction layer 
(the layer where there is net dissociation) extends into both the gel and resin domains. Since the thickness of the resin disc is much larger than the reaction layer in the gel domain, almost all metal accumulated originates from dissociation of the complex in the resin domain. Dissociation in the resin domain has allowed to justify the labile behaviour of the complex of Cd with nitrilotriacetic acid (NTA) [11] while, without penetration into the resin, it was expected to be only partially labile.

Interesting additional information on the resin role can be gained when DGT devices with two resin discs are used. Thus, the essentially perfect sink behaviour of the resin was tested in a work with only metals (i.e., in absence of ligands) and DGT devices with two or three resin discs [17]. This work demonstrated that a kinetic association constant between the metal and the resin sites could, in principle, be obtained from the distribution of the metal bound to two resin discs, whenever this distribution lies within a meaningful sensitive window of kinetic constants. In the presence of complexes, both, the metal accumulation and the distribution of the metal in the stack of resin discs have been used to fit the parameters of the system Ni-NTA $[15,18]$. Notice that the availability of complementary information to that of the total accumulation in DGT experiments is of high interest, since it helps in fitting the parameters or even allows the direct determination of some of them.

All these works, and the derived analytical expressions, assumed a homogeneous distribution of the resin sites in the resin domain. This assumption can be considered as a first approximation, since the resin beads partially settle during casting of the gel $[3,15]$. It seems, then, timely to check the influence of the inhomogeneous distribution of the resin sites in the resin disc by quantifying the effect on the total accumulation, on the distribution 
of metal accumulation in a stack of resin discs and on the accuracy of the fitted parameters obtained assuming homogeneous binding site distribution.

In this manuscript we consider that the binding beads appear only in half of the resin disc [15] (see a schematic depiction in Figures SI-1 and SI-2). The label R/2 will denote this resin disc (Figure SI-2), while $\mathrm{R}$ will denote the homogeneously distributed resin disc (Figure SI-1). Section 2 outlines the mathematical formulation and solution of this problem. Section 3 compares the performance of DGT devices containing one $\mathrm{R}$ or $\mathrm{R} / 2$ resin disc, and Section 4 compares the behaviour of DGT devices with a stack of two homogeneous or of two half-occupied resin discs.

\section{Theoretical model}

Let us consider a system that contains in solution a metal $\mathrm{M}$ and a ligand $\mathrm{L}$ which react as

$$
\mathrm{M}+\mathrm{L} \underset{k_{\mathrm{d}}}{\stackrel{k_{\mathrm{a}}}{\rightleftharpoons}} \mathrm{ML}
$$

where $k_{\mathrm{a}}$ and $k_{\mathrm{d}}$ are the association and dissociation rate constants, $K=\frac{c_{\mathrm{ML}}^{*}}{c_{\mathrm{M}}^{*} c_{\mathrm{L}}^{*}}$ is the corresponding stability constant, $K^{\prime}=\frac{c_{\mathrm{ML}}^{*}}{c_{\mathrm{M}}^{*}}$ is the effective stability constant, and $c_{i}^{*}$ is the concentration of species $i$ in the bulk solution. When a DGT device is introduced in this solution, all these species diffuse and react through the diffusive gel $(r<x<r+g)$ until reaching the resin domain $(0<x<r)$ where, due to the presence of the binding beads, another reaction takes place: 
$\mathrm{M}+\mathrm{R} \underset{k_{\mathrm{d}, \mathrm{R}}}{\stackrel{k_{\mathrm{a}, \mathrm{R}}}{\rightleftharpoons}} \mathrm{MR}$

The mathematical formulation of all these processes is given elsewhere $[11,12,14,19]$. A new simulation code allowing the numerical solution of these equations has been written using the Finite Element Method. A spatial grid with unequal distribution of spatial positions has been used, reducing CPU time and hardware requirements in comparison to algorithms based on Finite Differences.

The numerical solution for the concentration profiles can be used to calculate the flux of metal bound to the resin, $J$, as well as the metal accumulation, $n_{\mathrm{M}}$, both in $\mathrm{R}$ and $\mathrm{R} / 2$ devices. Details are given in the SI section 1.3.

In DGT practice, the most interesting cases are those where the association rate constant $k_{\mathrm{a}, \mathrm{R}}$ between the metal and the resin sites and the stability constant of the metal-resin complex are so high that the resin can be considered as a perfect sink for the metal. Analytical expressions of concentration profiles (restricted to these cases) are reported in the SI. Accordingly, we will consider as a good approximation the existence of zero metal concentration in the resin domain, whenever the accumulation is far from saturation, while L and ML undergo diffusion and reaction along both gels. The restriction to perfect sink, together with the assumption of ligand excess conditions leads to an approximate analytical solution for the concentration profiles of metal and complex, as given in the SI sections 1.1 and 1.2, for both $\mathrm{R}$ and $\mathrm{R} / 2$ devices.

In the case of $\mathrm{R}$ devices, the total accumulation at time $t$ becomes (see the SI section 1.3): 
$n_{\mathrm{M}}=t A k_{\mathrm{d}} \lambda_{\mathrm{ML}} \tanh \left(\frac{r}{\lambda_{\mathrm{ML}}}\right) c_{\mathrm{ML}}^{r}+t A D_{\mathrm{M}}\left(\frac{c_{\mathrm{M}}^{*}+\varepsilon c_{\mathrm{ML}}^{*}-\varepsilon c_{\mathrm{ML}}^{r}}{g\left(1+\varepsilon K^{\prime}\right)}+\frac{\varepsilon c_{\mathrm{ML}}^{r} \operatorname{coth}\left(\frac{g}{m}\right)}{m\left(1+\varepsilon K^{\prime}\right)}\right)$

where the value of the concentration of the complex at $x=r, c_{\mathrm{ML}}^{r}$, can be computed as:

$$
c_{\mathrm{ML}}^{r}=\frac{c_{\mathrm{ML}}^{*}\left(1+\varepsilon K^{\prime}\right)}{\varepsilon K^{\prime}+\frac{g}{m} \operatorname{coth}\left(\frac{g}{m}\right)+\frac{g\left(1+\varepsilon K^{\prime}\right)}{m \lambda_{\mathrm{ML}}} \tanh \left(\frac{r}{\lambda_{\mathrm{ML}}}\right)}
$$

$\varepsilon$ is the normalized diffusion coefficient of the metal complex:

$$
\varepsilon=\frac{D_{\mathrm{ML}}}{D_{\mathrm{M}}}
$$

$m$ is a distance related to the thickness of the layer within the diffusive gel where the metal and the complex are not in equilibrium:

$$
m=\sqrt{\frac{D_{\mathrm{ML}}}{k_{\mathrm{d}}\left(1+\varepsilon K^{\prime}\right)}}
$$

and the penetration parameter $\lambda_{\mathrm{ML}}$, can be calculated as:

$$
\lambda_{\mathrm{ML}}=\sqrt{\frac{D_{\mathrm{ML}}}{k_{\mathrm{d}}}}
$$

Complementary information can be gained when DGT devices with two resin discs are used. Metal accumulation in the back resin disc will indicate the presence of partially labile complexes that penetrate into the resin by diffusion. The percentage of the total mass that is accumulated in the back resin disc (for 2 disc stacks of $\mathrm{R}$ devices) [20] will be:

$$
\% \text { back }=\frac{m \varepsilon K^{\prime}}{2 m\left(1+\varepsilon K^{\prime}\right) \cosh \left(\frac{r}{2 \lambda_{\mathrm{ML}}}\right)+\lambda_{\mathrm{ML}} \cosh \left(\frac{r}{\lambda_{\mathrm{ML}}}\right) \operatorname{coth}\left(\frac{g}{m}\right) \operatorname{csch}\left(\frac{r}{2 \lambda_{\mathrm{ML}}}\right)}
$$


The contribution of complex dissociation in the diffusive gel is negligible for typical cases. In these cases, expression (8) becomes:

$\%$ back $=\frac{1}{2} \operatorname{sech}\left(\frac{r}{2 \lambda_{\mathrm{ML}}}\right)$

Details for the derivation of equations (8) and (9) are given in the SI section 1.3.

The lability degree

The lability degree $(\xi)$ [21] of a complex is a measure of the actual contribution of the complex to the metal accumulation in comparison to its possible maximum contribution. The lability degree takes values between 0 and 1 . For labile systems $(\xi=1)$, the kinetic processes are so fast that the metal and complex are in equilibrium at any relevant spatial position in the gel, except in a layer of negligible thickness at the resin disc-diffusive gel interface (the reaction layer in the diffusive gel). For inert systems $(\xi=0)$, dissociation of the complex is so slow that the complex concentration profile in the gel domain is flat [13]. Here the system does not reach equilibrium at any position in the diffusive gel. Partially labile complexes are between these two limits.

The lability degree can be calculated as [21, 22]

$\xi=\frac{J-J_{\text {free }}}{J_{\text {lab }}-J_{\text {free }}}=1-\frac{C_{\mathrm{ML}}^{r}}{C_{\mathrm{ML}}^{*}}$

so that, when there is full dissociation of the complex at $x=r, \xi=1$ and the complex is labile. For an inert complex $c_{\mathrm{ML}}^{r}=c_{\mathrm{ML}}^{*}$ and $\xi=0$. 
Experimentally, in systems with neglible flux of the free metal in comparison with that of the complex, the lability degree can be determined as [20]

$\xi \approx \frac{n_{\mathrm{M}}}{\varepsilon n_{\mathrm{M}}^{\text {no-ligand }}}$

where $n_{\mathrm{M}}$ is the accumulation in presence of ligand while $n_{\mathrm{M}}^{\text {no-ligand }}$ is the accumulation in absence of ligand, at the same total metal concentration.

\section{Results for DGT devices with one resin disc}

\subsection{Concentration profiles}

Normalized concentration profiles $\left(c_{i}(x) / c_{i}^{*}\right)$ for M, L and ML computed from numerical simulation and analytical solutions (as described in the SI sections 1.1 and 1.2) are shown in Figures 1 and 2 for cases where the complexation equilibrium constant takes the values $K=10 \mathrm{~m}^{3} \mathrm{~mol}^{-1}$ and $K=10^{3} \mathrm{~m}^{3} \mathrm{~mol}^{-1}$, respectively. The agreement between the analytical and numerical concentration profiles for these parameters is excellent and the curves are almost identical. For this reason only one single line for $\mathrm{M}$ and one for ML are shown in Figures 1 and 2. Free metal concentration profiles are almost zero along the resin domain for the R devices (see Figures 1a and 2a), in agreement with the fast and strong metal binding to the resin sites considered. Recalling that, when the total concentration of $\mathrm{M}$ is negligible in front of L (i.e.: inexcess of ligand), the divergence of the normalized metal and complex concentration profiles indicates disequilibrium between both species, i.e., net dissociation, Figures $1 \mathrm{a}$ and $2 \mathrm{a}$ indicate that the reaction layer in the $\mathrm{R}$ devices extends 
along the resin domain plus an extra layer in the gel domain whose thickness $m$ can be computed with equation (6). In the $\mathrm{R} / 2$ devices, the metal concentration is non-negligible in the volume region without binding sites (see Figures $1 \mathrm{~b}$ and $2 \mathrm{~b}$ ), as a consequence of the release of $\mathrm{M}$ by complex dissociation and the absence of binding agent in those regions. Under some conditions, this dissociation can be fast enough for the system $\mathrm{M}+\mathrm{L}$ to reach equilibrium, as shown in the leftmost part of Figure 2b.

There is an important influence of $K^{\prime}\left(K^{\prime}=K c_{\mathrm{L}}^{*}=\frac{c_{\mathrm{ML}}^{*}}{c_{\mathrm{M}}^{*}}\right)$ on the metal profiles (compare Figures $1 \mathrm{~b}$ and $2 \mathrm{~b}$ ), even when a fixed value of $k_{\mathrm{d}}$ is used. The expression for $m$ helps in justifying the influence of $K^{\prime}$ in Figures $1 \mathrm{~b}$ and 2b. Equation (6) indicates that when $K^{\prime}$ increases, thinner reaction layers are obtained, so that $m<r$ in Figure 2b, while $m>r$ in Figure $1 \mathrm{~b}$. In other words, small values of $m$ indicate that the dissociation of the complex is quite effective in buffering the metal consumption at $x=r / 2$. For instance, the normalized metal concentration increases and merges with the normalized complex concentration profile in a part of the layer without binding agent $(0<x<r / 2)$ in Figure $2 \mathrm{~b}\left(K^{\prime}=10^{3}\right)$ and thus, very little dissociation is expected in this volume.

\subsection{Influence of the inhomogeneous binding site distribution on the total metal accumulation}

Since larger differences in the concentration profiles were found for high values of $K$ ', $K^{\prime}=10^{3}$ was used in Figure 3 to analyse the influence of the homogeneity of the resin site distribution on the accumulation and lability degree of complexes measured in the DGT 
devices. As expected, the total accumulations in both DGT devices increase as the dissociation constants of the complexes increase. It also indicates that the accumulation for $\mathrm{R} / 2$ device is always less than for $\mathrm{R}$ device.

Moreover, the total accumulations in $\mathrm{R}$ and $\mathrm{R} / 2$ devices are very close for low or high enough values of $k_{\mathrm{d}}$, while they diverge for intermediate $k_{\mathrm{d}}$ values. As noted above, when the complex is very labile, the entire complex dissociates at the resin disc-diffusive gel interface $(x=r)$ so that the absence of reactive sites at the bottom of the resin layer is irrelevant with respect to the accumulation. A similar situation arises for inert complexes: dissociation is negligible (both in the gel or the resin layers) and the inhomogeneity of the binding bead distribution has no impact on the metal accumulation.

For intermediate values of $k_{\mathrm{d}}$, dissociation inside the resin disc is the main mechanism of metal accumulation. In this situation the inhomogeneous resin site distribution has the highest influence on metal accumulation.. A similar behaviour is observed for the lability degree in Figure 3b, which can also be understood using the same arguments as for the accumulation. In order to assess an extreme situation with a large discrepancy between results of $\mathrm{R}$ and those of $\mathrm{R} / 2$, calculations for a set of $K$ values by changing $k_{\mathrm{a}}$ and $k_{\mathrm{d}}$ were done. Table 1 shows the percentages of largest discrepancy between the accumulation and lability in $\mathrm{R}$ and $\mathrm{R} / 2$ devices. The maximum influence of the inhomogeneity of the resin disc increases as $K$ increases until the lability reaches an almost fixed value close to 0.25. Notice that this lability degree corresponds to a fixed $k_{\mathrm{d}}$ of the complex (see column 3 in Table 1) which indicates that, for $g>r$, the lability degree essentially depends on $k_{\mathrm{d}}$ but not on $k_{\mathrm{a}}$ and $K$, as stated in [12]. Although the decrease of accumulation in the $\mathrm{R} / 2$ devices 
increases as $K$ increases, it remains below $13 \%$ for $K^{\prime}=10^{2} \mathrm{~m}^{3} \mathrm{~mol}^{-1}$ (i.e. $K^{\prime}=10^{5} \mathrm{~L} \mathrm{~mol}^{-1}$ ) in the worst scenario (Table 1). Notice that stronger complexes could increase this error, but it should also be taken into account that such strong complexes will distort the linear accumulation in the DGT due to their similar (or higher) affinity for the metal to that of the resin [23].

\subsection{Influence of the system parameters on the difference between $R$ and $R / 2$ devices}

Metal complexes with simple ligands have diffusion coefficients close to those of the hydrated metal ions, since the sizes of both species are not very different. For macromolecular metal complexes like those with humic matter, the diffusion coefficients can be one order of magnitude smaller than the diffusion coefficient of the free metal ion [24].

Figure 4 shows the influence of the diffusion coefficient of the complex on the metal accumulation in $\mathrm{R}$ and $\mathrm{R} / 2$ devices. The accumulation decreases as $D_{\mathrm{ML}}$ decreases, since the transport of $\mathrm{M}$ from the solution to the resin domain decreases. The system is not fully labile since both, the $\mathrm{R}$ and $\mathrm{R} / 2$ curves are not linear as it would be expected for a labile

system $\left(n_{\mathrm{M}, \text { labile }}=A t \frac{D_{\mathrm{ML}} c_{\mathrm{ML}}^{*}}{g}\right.$ whenever $\left.\varepsilon K^{\prime}>>1\right)$. For fixed values of $K$ and $k_{\mathrm{d}}$, Figure 4 shows a decreasing divergence of the accumulation in the $\mathrm{R}$ and $\mathrm{R} / 2$ devices as the diffusion coefficient of the complex decreases, a result of interest when complexes with natural macromolecular ligands are present in the system. Actually, the increase of $D_{\mathrm{ML}}$ in 
Figure 4 is concomitant to an increase of the lability degree which approaches 0.25 , the value corresponding to the maximum influence of the inhomogeneous distribution of the reactive sites (see Table 1 ).

Increasing the resin layer thickness increases both the metal accumulation and the lability degree (data not shown). As explained elsewhere $[12,13]$, an increase of the resin thickness increases the volume where there is net dissociation. This is true whenever partially labile complexes are present in the system, can penetrate in the resin domain and they do not reach full dissociation in the resin domain. Divergences between $R$ and $R / 2$ devices decrease as the thickness of the resin layer decreases (e.g. in ultra-thin DGT [25]), until convergence to a common value for devices with negligible resin thickness, or whenever penetration of complexes was prevented. In the other limit, reduction of the differences between $\mathrm{R}$ and $\mathrm{R} / 2$ devices is also expected when the resin is thick enough to reach full dissociation of complexes in the layer $r / 2<x \leq r$.

\subsection{Inhomogeneity effects in commercial DGT devices when sampling metal availability in natural waters}

Commercial DGT devices use a binding-bead concentration in large excess to avoid saturation effects under typical working conditions. Thus, small fluctuations in the resin distribution do not influence the accumulation. However, it is well known that the resin beads of Chelex 100 tend to settle to one side of the resin disc while casting. The average diameter of these beads is $100 \mu \mathrm{m}$. The amount of resin beads used per disc is close to that corresponding to a packed single layer of beads at the surface of the resin disc, as 
schematically depicted in [3]. If the settling was total, only $1 / 4$ of the resin volume would contain resin beads. An imperfect settling renders reasonable that around half of the volume of the resin disc may contain Chelex beads in a standard resin disc (thickness $400 \mu \mathrm{m}$ ). Thus, results reported in this work can be straightforwardly applied to commercial DGT devices.

Table 1 indicates the highest percentage of decrease in the accumulation due to settling for different values of the stability constant. Notice that this percentage applies only to the contribution of partially labile complexes, while free metal and labile complexes are not influenced by the settling of the resin. Assuming Eigen ideas (i.e. a fixed $k_{\mathrm{a}}$ ), the lability decreases as the stability of the complex increases. As a rule of thumb, inorganic complexes tend to be labile in DGT so that the main influence of the resin settling is mostly linked to complexes with organic ligands. Some strong organic metal complexes, partially labile in DGT, have stabilities in the range of the values reported in Tables 1 and 2. For instance, Visual MINTEQ uses a stability constant for NiNTA at $25^{\circ} \mathrm{C}$ of $\log K=9.39$ and DGT experiments in systems with $\mathrm{c}_{T, N i}=0.01 \mathrm{~mol} \mathrm{~m}^{-3}$ and $\mathrm{c}_{T, N T A}=1.0 \mathrm{~mol} \mathrm{~m}{ }^{-3}$ indicate that NiNTA behaves as partially labile with $\log K^{\prime}=6,3$. NTA can be found in natural waters due to anthropogenic activities [26], and it is also studied as model of natural organic matter. Thus, metal accumulation from such complexes could also be influenced by the settling of the resin in the DGT although other phenomena like accumulation of humic acid on the diffusive gel have also been described [27]. In any case, the reduction of accumulated mass due to the resin settling is always below the percentage of volume of the resin domain without resin beads (50\%), since part of the metal released there still binds to the resin after travelling back by diffusion. 


\section{DGT devices with two resin discs}

The use of DGT devices with a stack of resin discs has been suggested as a way of obtaining complementary information on the behaviour of the system $[15,17,18]$. By eluting and analysing each resin disc separately, these devices allow assessing whether the resin acts as a perfect sink for the free metal. The condition of perfect sink is necessary to support the application of the simplest formula to describe the metal accumulation in DGT, i.e.:

$$
c_{\mathrm{DGT}}=\frac{n_{\mathrm{M}} g}{A t D_{\mathrm{M}}}
$$

The use of disc stacks can also help to elucidate if complexes are partially labile and can penetrate into the resin domain. Recently, it has been highlighted that the knowledge of the metal distribution in the front and back resin discs can be used to fit the kinetic dissociation constant of the complex in the resin domain $[18,20] .$. For all these reasons, it is of interest to assess the influence of the inhomogeneity of the binding sites within the resin disc on the accumulation, lability degree of the complex, distribution of the accumulated mass (between front and back resin discs) and the retrieval of the kinetic dissociation constant from this distribution.

DGT devices with two homogeneous (R) or two half occupied resin discs (R/2) were studied by numerical simulation. Standard resin discs of $4 \times 10^{-4} \mathrm{~m}$ thickness and diffusive gel of $8.52 \times 10^{-4} \mathrm{~m}$ thickness were considered. Figure 5 shows very different concentration

profiles of metal for both types of resin discs. The physical explanation outlined in Section 3.1 can be extended here to rationalize this behaviour. 
Parallel to Table 1, Table 2 reports (for each $K$ value) the metal accumulations in DGT devices that accommodate two $\mathrm{R}$ or two $\mathrm{R} / 2$ resin discs. Only data corresponding to the kinetic constants that lead to the maximum discrepancies between both devices are reported. The lability degree of complexes obtained in Table 2 is quite constant, but higher than the lability degree of the complexes reported in Table 1 for just one resin disc. The maximum discrepancy between both types of resins (Table 2) arises for complexes with lability degree below 0.5 . As in the case with only one resin device, the maximum discrepancy increases as $K$ increases, but the relative difference remains lower than the fraction of volume without binding agent.

DGT devices with two resin discs can be used to find $k_{\mathrm{d}}$ of the complex by fitting the $\%$ of back accumulation using the analytical expression (8) or, alternatively, by fitting the total accumulation with the analytical expression (3). In order to check the influence of the inhomogeneous distribution of the binding beads on the recovered $k_{\mathrm{d}}$ values, numerical simulations of DGT devices with two $\mathrm{R} / 2$ resin discs with partially labile complex in excess of ligand were conducted. These were used to compute the \% back and total accumulation. Subsequently these values were used to recover $k_{\mathrm{d}}$ using the analytical expressions (3) or (8). The results are reported in Table 3.

$k_{\mathrm{d}}$ values fitted from \%back are of the same order of magnitude than the one used in the simulation $\left(10^{-2} \mathrm{~s}^{-1}\right)$, but systematically modified by a factor close to $1 / 2$ (i. e., recovered values tend to be half of the correct ones). The agreement of the values recovered from the total accumulation is better, with relative errors around $25 \%$. The consistency of these 
errors for all the $K$ values examined suggests the use of this empirical factor as a way to improve the estimation.

To check the applicability of this correction factor, new simulations in DGT devices with two $\mathrm{R} / 2$ resin discs were conducted for systems with a fixed equilibrium constant $\left(K=10^{4}\right.$ $\mathrm{m}^{3} / \mathrm{mol}$ ) and with values of $k_{\mathrm{d}}$ scanning the range of lability degrees from inert complexes to labile complexes. Results are listed in Table 4, which confirms that the errors in recovering $k_{\mathrm{d}}$ from the \%back data assuming a homogeneous distribution of the resin beads are also quite independent of the actual $k_{\mathrm{d}}$. The simulated values indicate that a factor of 2 is suitable to improve the value of $k_{\mathrm{d}}$ (assuming homogeneous distributions of the binding agent) estimated from \%back measurement. For other fractions $(f)$ of volume in the resin disc being occupied by the binding agent, a better estimate of $k_{\mathrm{d}}$ is $f^{-1} k_{\mathrm{d} \text {,recovered, when data }}$ from \%back is used (data not shown). In the case of the values recovered using the total accumulation as input information, the error depends on $k_{\mathrm{d}}$, so one cannot suggest a general correction factor .

Notice, however, that the accuracy decreases for both low and high \% back, especially if we also include the experimental measurement uncertainty, which is not considered here. Actually, for a given thickness of the resin disc there is a window of sensitive $k_{\mathrm{d}}$ values [20]. Too high $k_{\mathrm{d}}$ values lead to negligible accumulation in the back resin disc, since the complex has been fully dissociated in the diffusive gel and the adjacent resin disc. Conversely, $k_{\mathrm{d}}$ values below a given threshold lead to a \% back close to $50 \%$ and thus no information on the decrease of the complex concentration along the resin domain is 
obtained. Therefore, only intermediate values of \% back contain significant information on the complex dissociation [20].

\section{Conclusions}

Analytical expressions for the concentration profiles in an inhomogeneous resin layer have been derived and their predictions agree with the rigorous numerical simulations (see Figures 1 and 2). For very labile or inert complexes, the distribution of binding beads in the resin layer does not show influence on the concentration profiles, on the total accumulation of metal and on the lability degree of complexes. For partially labile complexes, the influence of the spatial distribution of binding beads increases with the value of the equilibrium constant $K^{\prime}$ (see Table 1) and the diffusion coefficient of the complex (see Figure 4). In these cases, the assumption of binding site homogeneity always leads to underestimation of both $c_{\mathrm{DGT}}$ and the accumulation, but the present results indicate that only $13 \%$ decrease of accumulation arises in both i) inhomogeneous devices where resin beads are only dispersed in half of the resin volume and ii) complexes with stability constant $K^{\prime}<10^{5} \mathrm{~L} \mathrm{~mol}^{-1}$ dominating the metal speciation.

The complex dissociation rate constant, $k_{\mathrm{d}}$, can be determined from the percentage of back accumulation in a DGT with a stack of two resin discs (section 4). This determination is a direct measurement of the kinetic dissociation constant in the resin domain, which can differ from the value in the diffusive gel or bulk solution as has recently been suggested [18]. However, the application of this procedure is restricted to a meaningful window of kinetic constants values [20]. The relative error values (due to inhomogeneity of the resin 
layer) found in recovering $k_{\mathrm{d}}$ from the \%back are quite independent of $K$ and $k_{\mathrm{d}}$. For $\mathrm{R} / 2$ devices there is a ratio of 2 between the real value and the recovered one assuming homogeneous distributions of binding resin beads.

\section{Acknowledgments}

Financial support from the Spanish Ministry of Education and Science (Projects CTM2012-

39183 and CTM2013-48967) is acknowledged. MJP acknowledges support from UdL and AA from MINECO BES-2013-065173.

\section{References}

1. W. Davison, H. Zhang, In-situ speciation measurements of trace components in naturalwaters using thin-film gels, Nature, 367 (1994) 546-548.

2. W. Davison, H. Zhang, Progress in understanding the use of diffusive gradients in thin films (DGT) - back to basics, Environ. Chem., 9 (2012) 1-13.

3. H. Zhang, W. Davison, Performance-characteristics of diffusion gradients in thin- films for the in-situ measurement of trace-metals in aqueous- solution., Anal. Chem., 67 (1995) 33913400.

4. H. Zhang, W. Davison, B. Knight, S. McGrath, In situ measurements of solution concentrations and fluxes of trace metals in soils using DGT, Environ. Sci. Technol., 32 (1998) 704-710.

5. H. Zhang, W. Davison, R. Gadi, T. Kobayashi, In situ measurement of dissolved phosphorus in natural waters using DGT, Anal. Chim. Acta, 370 (1998) 29-38.

6. O. Clarisse, B. Dimock, H. Hintelmann, E. P. H. Best, Predicting Net Mercury Methylation in Sediments Using Diffusive Gradient in Thin Films Measurements, Environ. Sci. Technol., 45 (2011) 1506-1512.

7. S. Tandy, S. Mundus, H. Zhang, E. Lombi, J. Frydenvang, P. E. Holm, S. Husted, A new method for determination of potassium in soils using diffusive gradients in thin films (DGT), Environ. Chem., 9 (2012) 14-23. 
8. Y. L. Zhang, S. Mason, A. McNeill, M. J. McLaughlin, Application of the diffusive gradients in thin films technique for available potassium measurement in agricultural soils: Effects of competing cations on potassium uptake by the resin gel, Anal. Chim. Acta, 842 (2014) 27-34.

9. C. E. Chen, H. Zhang, K. C. Jones, A novel passive water sampler for in situ sampling of antibiotics, Journal of Environmental Monitoring, 14 (2012) 1523-1530.

10. C. E. Chen, H. Zhang, G. G. Ying, K. C. Jones, Evidence and Recommendations to Support the Use of a Novel Passive Water Sampler to Quantify Antibiotics in Wastewaters, Environ. Sci. Technol., 47 (2013) 13587-13593.

11. S. Mongin, R. Uribe, J. Puy, J. Cecilia, J. Galceran, H. Zhang, W. Davison, Key Role of the Resin Layer Thickness in the Lability of Complexes Measured by DGT, Environ. Sci. Technol., 45 (2011) 4869-4875.

12. R. Uribe, S. Mongin, J. Puy, J. Cecilia, J. Galceran, H. Zhang, W. Davison, Contribution of Partially Labile Complexes to the DGT Metal Flux, Environ. Sci. Technol., 45 (2011) 53175322.

13. J. Puy, R. Uribe, S. Mongin, J. Galceran, J. Cecilia, J. Levy, H. Zhang, W. Davison, Lability Criteria in Diffusive Gradients in Thin Films, J. Phys. Chem. A, 116 (2012) 6564-6573.

14. N. J. Lehto, W. Davison, H. Zhang, W. Tych, An evaluation of DGT performance using a dynamic numerical model, Environ. Sci. Technol., 40 (2006) 6368-6376.

15. M. R. Shafaei-Arvajeh, N. Lehto, O. A. Garmo, H. Zhang, Kinetic Studies of Ni Organic Complexes Using Diffusive Gradients in Thin Films (DGT) with Double Binding Layers and a Dynamic Numerical Model, Environ. Sci. Technol., 47 (2013) 463-470.

16. R. Uribe, J. Puy, J. Cecilia, J. Galceran, Kinetic Mixture Effects in Diffusion Gradients in Thin Films (DGT), Phys. Chem. Chem. Phys., 15 (2013) 11349-11355.

17. J. L. Levy, H. Zhang, W. Davison, J. Puy, J. Galceran, Assessment of trace metal binding kinetics in the resin phase of diffusive gradients in thin films, Anal. Chim. Acta, 717 (2012) 143-150.

18. J. Puy, J. Galceran, S. Cruz-Gonzalez, C. A. David, R. Uribe, C. Lin, H. Zhang, W. Davison, Metal accumulation in DGT: Impact of ionic strength and kinetics of dissociation of complexes in the resin domain, Anal. Chem., 86 (2014) 7740-7748.

19. M. H. Tusseau-Vuillemin, R. Gilbin, M. Taillefert, A dynamic numerical model to characterize labile metal complexes collected with diffusion gradient in thin films devices, Environ. Sci. Technol., 37 (2003) 1645-1652.

20. Galceran, J. and Puy, J., Interpretation of diffusion gradients in thin films (DGT) measurements: a systematic approach, Environmental Chemistry, In press (2014). 
21. J. Galceran, J. Puy, J. Salvador, J. Cecília, H. P. van Leeuwen, Voltammetric lability of metal complexes at spherical microelectrodes with various radii, J. Electroanal. Chem., 505 (2001) 85-94.

22. F. Degryse, E. Smolders, H. Zhang, W. Davison, Predicting availability of mineral elements to plants with the DGT technique: a review of experimental data and interpretation by modelling, Environ. Chem., 6 (2009) 198-218.

23. S. Mongin, R. Uribe, C. Rey-Castro, J. Cecilia, J. Galceran, J. Puy, Limits of the Linear Accumulation Regime of DGT Sensors, Environ. Sci. Technol., 47 (2013) 10438-10445.

24. J. Balch, C. Gueguen, Effects of molecular weight on the diffusion coefficient of aquatic dissolved organic matter and humic substances, Chemosphere, 119 (2015) 498-503.

25. N. J. Lehto, W. Davison, H. Zhang, The use of ultra-thin diffusive gradients in thin-films (DGT) devices for the analysis of trace metal dynamics in soils and sediments: a measurement and modelling approach, Environ. Chem., 9 (2012) 415-423.

26. C. K. Schmidt, M. Fleig, F. Sacher, H. E. Brauch, Occurrence of aminopolycarboxylates in the aquatic environment of Germany, Environ. Pollut, 131 (2004) 107-124.

27. P. L. R. van der Veeken, H. P. van Leeuwen, DGT/DET Gel Partition Features of Humic Acid/Metal Species, Environ. Sci. Technol., 44 (2010) 5523-5527.

\section{Tables}


Table 1. Discrepancies in lability degree $(\xi)$ and total accumulation $\left(n_{\mathrm{M}}\right)$ between a DGT device with a homogeneous resin (R) and another DGT device with an inhomogeneous disc where resin beads are in just in one half of the volume (R/2). Parameters: $\mathrm{c}_{T, M}=0.01 \mathrm{~mol} \mathrm{~m}^{-3}, \mathrm{c}_{T, L}=1.0 \mathrm{~mol} \mathrm{~m}^{-3}, D_{\mathrm{M}}=7.07 \times 10^{-10} \mathrm{~m}^{2} \mathrm{~S}^{-}$ $1, D_{\mathrm{ML}}=4.95 \times 10^{-10} \mathrm{~m}^{2} \mathrm{~s}^{-1}$ and $t=24 \mathrm{~h}$.

\begin{tabular}{|c|c|c|c|c|c|c|}
\hline$K\left(\mathrm{~m}^{3} \mathrm{~mol}^{-1}\right)$ & $\begin{array}{c}k_{\mathrm{a}}\left(\mathrm{m}^{3} \mathrm{~mol}^{-1} \mathrm{~s}^{-}\right. \\
\left.{ }^{1}\right)\end{array}$ & $\left.k_{\mathrm{d}} \mathrm{s}^{-1}\right)$ & $\begin{array}{c}\xi \\
(\mathrm{R})\end{array}$ & $\begin{array}{c}n_{\mathrm{M}}(\mathrm{nmol}) \\
(\mathrm{R})\end{array}$ & $\begin{array}{c}\text { Discrepancy } \xi \\
(\%)\end{array}$ & $\begin{array}{c}\text { Discrepancy } n_{\mathrm{M}} \\
(\%)\end{array}$ \\
\hline 10 & $5 \times 10^{-3}$ & $5 \times 10^{-4}$ & 0.34 & 54.8 & 1.8 & 1.5 \\
\hline $10^{2}$ & $5 \times 10^{-2}$ & $5 \times 10^{-4}$ & 0.29 & 43.0 & 12.2 & 13.1 \\
\hline $10^{3}$ & $5 \times 10^{-1}$ & $5 \times 10^{-4}$ & 0.26 & 39.7 & 28.8 & 29.9 \\
\hline $10^{4}$ & 5 & $5 \times 10^{-4}$ & 0.25 & 38.4 & 41.1 & 41.2 \\
\hline
\end{tabular}

Discrepancy $\xi=\frac{\xi(R)-\xi(R / 2)}{\xi(R)}$; Discrepancy $n_{\mathrm{M}}=\frac{n_{\mathrm{M}}(R)-n_{\mathrm{M}}(R / 2)}{n_{\mathrm{M}}(R)}$

Table 2. Lability degree and total accumulation in DGT devices with two $\mathrm{R}$ or two R/2 resin discs.

Parameters as in Table 1.

\begin{tabular}{|c|c|c|c|c|c|c|c|c|}
\hline $\begin{array}{c}K \\
\left(\mathrm{~m}^{3} \mathrm{~mol}^{-}\right. \\
\left.{ }^{1}\right)\end{array}$ & $\begin{array}{c}k_{\mathrm{a}}\left(\mathrm{m}^{3} \mathrm{~mol}^{-}\right. \\
\left.{ }^{1} \mathrm{~s}^{-1}\right)\end{array}$ & $\begin{array}{c}k_{\mathrm{d}} \\
\left(\mathrm{s}^{-1}\right)\end{array}$ & $\begin{array}{c}\xi \\
(\mathrm{R})\end{array}$ & $\begin{array}{c}n_{\mathrm{M}}(\mathrm{nmol}) \\
(\mathrm{R})\end{array}$ & $\begin{array}{c}\% \text { back } \\
(\mathrm{R})\end{array}$ & $\begin{array}{c}\xi \\
(\mathrm{R} / 2)\end{array}$ & $\begin{array}{c}n_{\mathrm{M}} \\
(\mathrm{nmol}) \\
(\mathrm{R} / 2)\end{array}$ & $\begin{array}{c}\% \text { back } \\
(\mathrm{R} / 2)\end{array}$ \\
\hline 10 & $5 \times 10^{-3}$ & $5 \times 10^{-4}$ & 0.44 & 72.0 & 33.3 & 0.43 & 70.2 & 40.1 \\
\hline $10^{2}$ & $5 \times 10^{-2}$ & $5 \times 10^{-4}$ & 0.40 & 64.0 & 40.8 & 0.38 & 55.4 & 49.2 \\
\hline $10^{3}$ & $5 \times 10^{-1}$ & $5 \times 10^{-4}$ & 0.38 & 62.2 & 44.1 & 0.31 & 48.4 & 47.7 \\
\hline $10^{7}$ & $5 \times 10^{3}$ & $5 \times 10^{-4}$ & 0.36 & 56.2 & 46.1 & 0.24 & 37.4 & 47.9 \\
\hline $10^{9}$ & $5 \times 10^{5}$ & $5 \times 10^{-4}$ & 0.36 & 56.2 & 46.1 & 0.24 & 37.4 & 47.9 \\
\hline
\end{tabular}

Table 3. Recovered kinetic dissociation constant of a complex species when data from two R/2 devices are interpreted with the analytical expressions for homogeneous resins (equations 3 and 8).

Simulations run with $k_{\mathrm{d}}=10^{-2} \mathrm{~s}^{-1}$. The rest of parameters as in Table 1.

\begin{tabular}{|c|c|c|c|c|}
\hline $\begin{array}{c}K \\
\left(\mathrm{~m}^{3} \mathrm{~mol}^{-1}\right)\end{array}$ & $\%$ back & $n_{\mathrm{M}} \quad(\mathrm{mol})$ & $\begin{array}{c}k_{\mathrm{d}}\left(\mathrm{s}^{-1}\right) \\
\text { Recovered } \\
\text { from \% back }\end{array}$ & $\begin{array}{c}\left.k_{\mathrm{d}} \mathrm{s}^{-1}\right) \\
\text { Recovered } \\
\text { from } n_{\mathrm{M}}\end{array}$ \\
\hline $10^{2}$ & $26.2 \%$ & $1.20 \times 10^{-7}$ & $4.80 \times 10^{-3}$ & $6.85 \times 10^{-3}$ \\
\hline $10^{3}$ & $26.3 \%$ & $1.21 \times 10^{-7}$ & $4.85 \times 10^{-3}$ & $7.46 \times 10^{-3}$ \\
\hline $10^{4}$ & $26.3 \%$ & $1.21 \times 10^{-7}$ & $4.83 \times 10^{-3}$ & $7.50 \times 10^{-3}$ \\
\hline $10^{5}$ & $26.3 \%$ & $1.21 \times 10^{-7}$ & $4.83 \times 10^{-3}$ & $7.46 \times 10^{-3}$ \\
\hline
\end{tabular}

Table 4. Recovered kinetic dissociation constants from simulated data of two R/2 resin discs, with $K=10^{4} \mathrm{~m}^{3} \mathrm{~mol}^{-1}$. The rest of parameters as in Table 1 .

\begin{tabular}{|c|c|c|c|c|}
\hline$k_{\mathrm{d}}$ & \% back & $n_{\mathrm{M}}(\mathrm{mol})$ & $k_{\mathrm{d}}\left(\mathrm{s}^{-1}\right)$ & $k_{\mathrm{d}}\left(\mathrm{s}^{-1}\right)$ \\
\hline
\end{tabular}




\begin{tabular}{|c|c|c|c|c|}
\hline$\left(\mathrm{s}^{-1}\right)$ & & $\begin{array}{c}\text { Recovered } \\
\text { from \% back }\end{array}$ & $\begin{array}{c}\text { Recovered } \\
\text { from } n_{\mathrm{M}}\end{array}$ \\
\hline $5.0 \times 10^{-4}$ & 47.9 & $3.76 \times 10^{-8}$ & $2.45 \times 10^{-4}$ & $2.51 \times 10^{-4}$ \\
\hline $1.0 \times 10^{-3}$ & 46.1 & $5.84 \times 10^{-8}$ & $4.99 \times 10^{-4}$ & $5.18 \times 10^{-4}$ \\
\hline $5.0 \times 10^{-3}$ & 35.0 & $1.07 \times 10^{-7}$ & $2.47 \times 10^{-3}$ & $3.25 \times 10^{-3}$ \\
\hline $1.0 \times 10^{-2}$ & 26.4 & $1.21 \times 10^{-7}$ & $4.83 \times 10^{-3}$ & $7.50 \times 10^{-3}$ \\
\hline $3.0 \times 10^{-2}$ & 12.1 & $1.35 \times 10^{-7}$ & $1.36 \times 10^{-2}$ & $2.43 \times 10^{-2}$ \\
\hline $5.0 \times 10^{-2}$ & 6.7 & $1.40 \times 10^{-7}$ & $2.26 \times 10^{-2}$ & $4.31 \times 10^{-2}$ \\
\hline $1.0 \times 10^{-1}$ & 2.4 & $1.45 \times 10^{-7}$ & $4.25 \times 10^{-2}$ & $9.03 \times 10^{-2}$ \\
\hline $3.0 \times 10^{-1}$ & 0.2 & $1.51 \times 10^{-7}$ & $1.18 \times 10^{-1}$ & $3.57 \times 10^{-1}$ \\
\hline
\end{tabular}




\section{Figures}

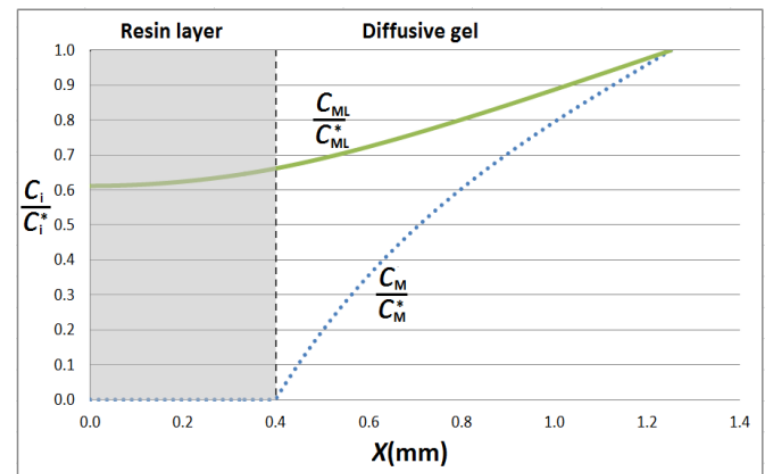

(a)

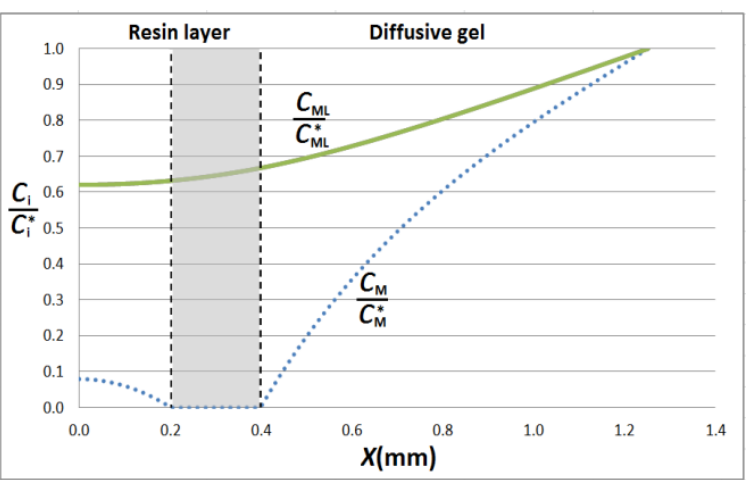

(b)

Figure 1. Normalized concentration profiles of metal and complex species for (a) $R$ resin (beads homogeneously distributed) and (b) $R / 2$ resin (beads in one half of the resin disc), with $K=10 \mathrm{~m}^{3} \mathrm{~mol}^{-1}, k_{\mathrm{d}}=$ $5 \times 10^{-4} \mathrm{~s}^{-1}, c_{\mathrm{T}, \mathrm{M}}=0.01 \mathrm{~mol} \mathrm{~m}^{-3}, c_{\mathrm{T}, \mathrm{L}}=1.0 \mathrm{~mol} \mathrm{~m}^{-3}, D_{\mathrm{M}}=7.07 \times 10^{-10} \mathrm{~m}^{2} \mathrm{~s}^{-1}, D_{\mathrm{ML}}=4.95 \times 10^{-10} \mathrm{~m}^{2} \mathrm{~s}^{-1}$ and $t=24 \mathrm{~h}$.

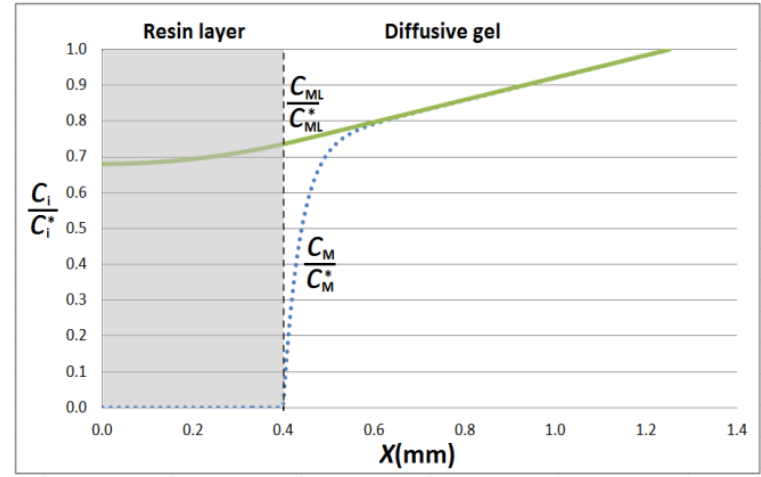

(a)

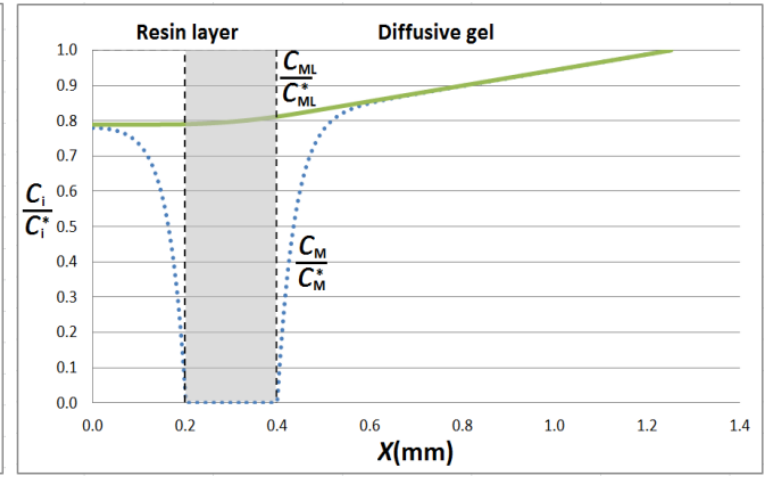

(b)

Figure 2. Normalized concentration profiles of metal and complex species for (a) $R$ resin and (b) $R / 2$ resin, with $\mathrm{K}=10^{3} \mathrm{~m}^{3} \mathrm{~mol}^{-1}$. The rest of parameters as in figure 1. 


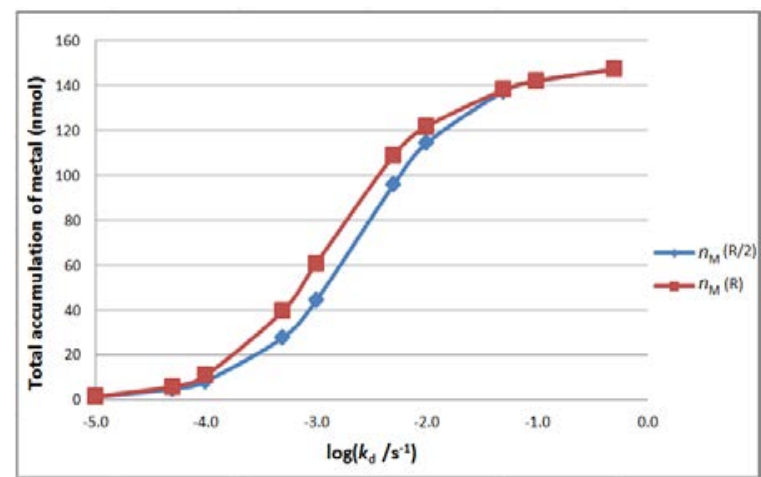

(a)

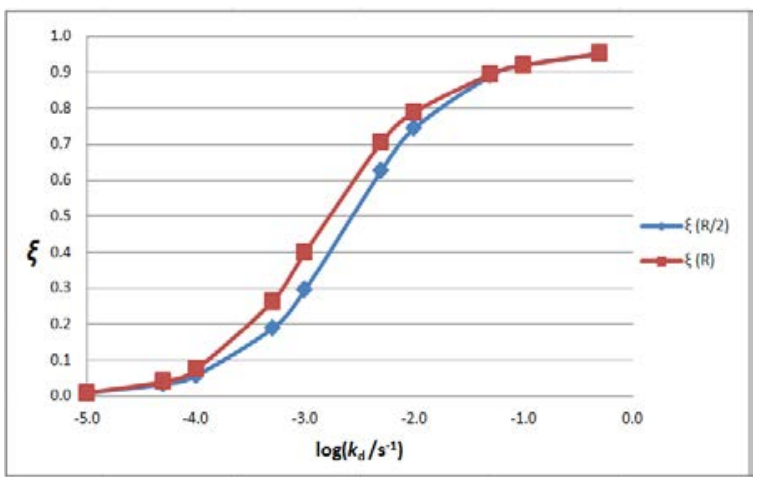

(b)

Figure 3. (a) Total accumulation of metal and (b) Lability degree of the complex as functions of $k_{\mathrm{d}}$. $K=10^{3}$ $\mathrm{m}^{3} \mathrm{~mol}^{-1}, \mathrm{r}=0.4 \mathrm{~mm}$. The rest of parameters as in figure 1. Markers: Red square for $R$ device, blue diamond for $R / 2$ device. Lines represent a guide to the eye.

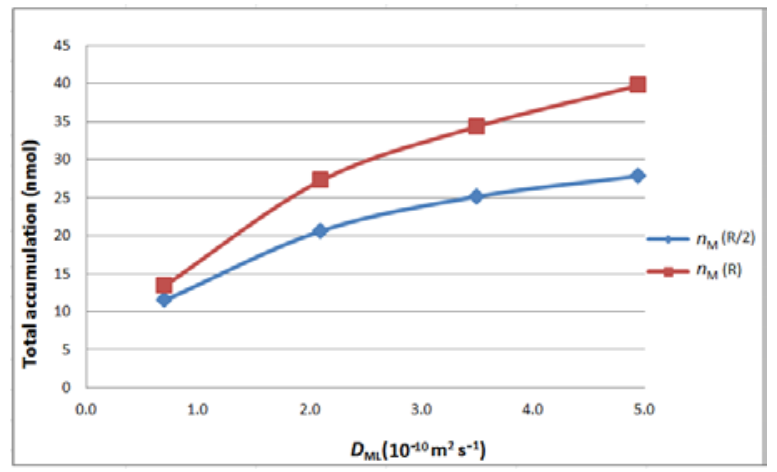

Figure 4. Total accumulation as a function of the diffusion coefficient of the complex. $D_{\mathrm{ML}}=0.7 \times D_{\mathrm{M}}$. The rest of parameters as in figure 3. Markers and lines as in previous figure.

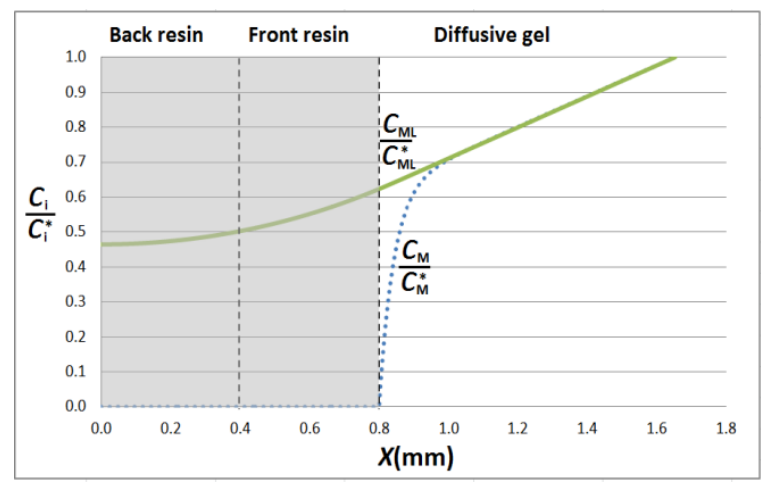

(a)

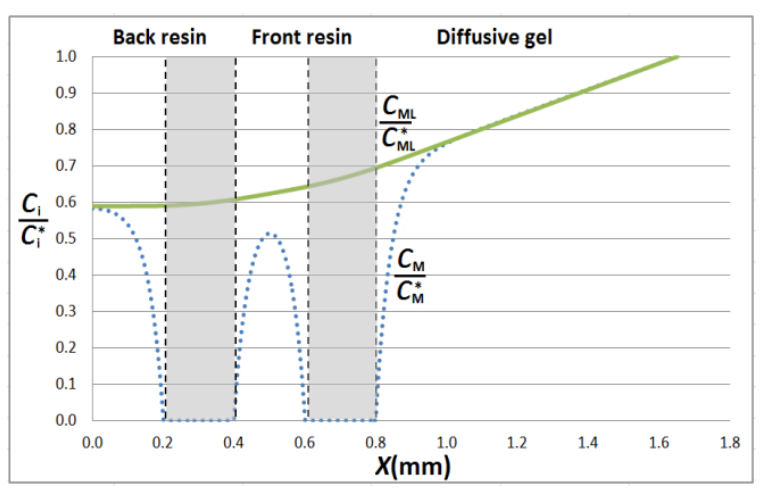

(b)

Figure 5. Normalized concentration profiles of species for (a) two $R$ resins and (b) two $R / 2$ resins. The rest of parameters as in figure 2. 